\title{
Pensamiento, idea e ideatum en la filosofía de Spinoza
}

\author{
Thought, idea and ideatum \\ in the philosophy of Spinoza
}

DANIEL ÁLVAREZ MONTERO

Universidad de La Laguna, España

Recibido: 26/04/2016 Aceptado: 24/11/2016

\section{RESUMEN:}

Un problema recurrente de la filosofía spinozista ha sido la pregunta por el estatus de los atributos en torno a su consideración respecto de la Sustancia. Su equidad es condición necesaria del sistema y, en virtud de ello, deben ser representables sólo en términos de sí mismos. A partir de los atributos de la extensión y el pensamiento, en este artículo enfocaré la inveterada problemática en torno a la aparente mayor extensión del atributo del pensamiento, esbozando una interpretación del mismo que permite entenderlo y expresarlo en los términos que exige el modelo del autor hispanoportugués.

\section{PALABRAS CLAVE}

SPINOZA, ATRIBUTOS, IDEA, PENSAMIENTO, CONCIENCIA.

\begin{abstract}
:
A recurring problem of the philosophy of Spinoza has been the question about the status of the attributes and its consideration in relation to the one Substance. Their equality is a necessary condition of the system and each attribute have to be representable only in terms of itself. From the attributes of Thought and Extension, in this paper I will focus on the rooted difficulties around the apparent greater extension of the attribute of Thought, sketching an interpretation that allows us to understand and express it in the terms that the spinozistic model demands.

KEYWORDS
\end{abstract}

SPINOZA, ATTRIBUTES, IDEA, THOUGHT, CONSCIOUSNESS.

(C) Contrastes. Revista Internacional de Filosofia, vol. XXII-Nº2 (2017), pp. 77-90. ISSN: 1136-4076

Departamento de Filosofía, Universidad de Málaga, Facultad de Filosofía y Letras Campus de Teatinos, E-29071 Málaga (España) 


\section{INTRODUCCIÓN}

El paralelismo spinozista asume, como premisa fundamental, que cuanto sea predicable de un atributo, debe ser también predicable de otro. En lo relativo a lo humano -el ejemplo sistémico de esta filosofía-, mediante su disposición y capacitación para percibir dos de los infinitos atributos, esta respectividad paralela debe hacerse notoria entre los atributos de la extensión y el pensamiento. No obstante, los siglos de lecturas han mostrado una prolífica serie de controversias en torno a cómo es posible extrapolar, sin perjuicio, lo predicable del primero en términos del segundo. La problemática es muy simple: el pensamiento, además de pensarse a sí mismo - esto es, ser consciente, en el caso del humano, de su propio pensar-, tiene por contenido ideas de objetos, de modos de otros atributos - para el presente caso, de la extensión-, y en este sentido parece transgredir sus propios límites, cosa que no puede suceder porque Spinoza explicita justo lo contrario: «Cada atributo de una misma substancia debe concebirse por sí.» ${ }^{1}$

La cuestión en torno a la mayor extensión del atributo del pensamiento lastra un problema a mi juicio mayor: la crítica $-\mathrm{O}$, al menos, su planteamiento general-, en torno a la infinitud de los atributos, que gira alrededor de lo que considero -y esto ha sido dicho con anterioridad, como se verá enseguida- una mala interpretación y extrapolación de una metáfora espacial sólo concebible desde la perspectiva de la extensión, puesto que del lado del pensamiento toda concepción de extensión o de aprehensión en tales términos carecería de sentido. Harold $\mathrm{H}$. Joachim, hace más de cien años, se percató de este planteamiento capcioso del problema, según señala muy brevemente en su estudio de la Ética que: «Toda la crítica [de ese aparente problema de la extensión del atributo del pensamiento], descansa en el abuso de una metáfora espacial. Los atributos están 'en' Dios, pero Dios no es un todo espacial, incluso cuando la extensión es uno de sus atributos.»²

Aseveraciones en principio laxas como, por ejemplo, aquélla de que el atributo del pensamiento abarca otros atributos -en la medida en que puede hacerlos objetos de ideas, caso cotejado con la extensión - arrastra ya consigo el error de una extensión del pensamiento, puesto que en el ámbito de las ideas toda consideración de cercanía o amplitud es mera retórica imaginativa, y lo

1 E, I, 10. Sigo, tanto para esta cita a la Ética como para las siguientes en este artículo, la traducción de Vidal Peña en Ética, Alianza, Madrid, 2006

2 Harold H. Joachim, A Study of the Ethics of Spinoza, Oxford Clarendon Press, Londres, 1901, p. 136. 
más que podría predicarse es un orden lógico. El ideatum de cada idea puede ser un modo de cualquier otro atributo, pero esto no lo convierte en parte de la idea, o se ve absorbido por ella, como si dos entidades diferentes fueran. El pensamiento es otra expresión de la Sustancia, lo que conlleva asumir el paralelismo spinozista tal y como creo que debe ser expresado y comprendido -y otros lo han señalado mucho antes ${ }^{3}-$ : como una relación de identidad entre atributos, que sólo son diferenciables, como afirmó en repetidas ocasiones el propio autor, en relación al intelecto. ${ }^{4}$

No obstante, la ambigüedad surge al considerar la naturaleza del atributo del pensamiento en torno a su posibilidad de objetivar y objetivarse a sí mismo - esto es, no sólo en términos de identidad modo de la extensión-modo del pensamiento, sino en cuanto idea que se vuelve sobre sí misma, objetivándose-. En este sentido, el ideatum de cada idea constituiría el propio objeto expresado en cuanto idea, no hay un puente o relación entre la propia idea y el objeto, pues son lo mismo: «Así también, un modo de la extensión y la idea de dicho modo son una sola y misma cosa, pero expresada de dos maneras. ${ }^{5}$

Las ideas de ideas, un tema también tratado respecto de la filosofía de Spinoza, así como las ideas de ideas de ideas -y así hasta el infinito, si se quiere-, podrían suponer un problema a esta concepción en la medida en que, o se asume que una idea de una idea es real sólo en cuanto pensamiento -y no tendría expresión en términos de la extensión, lo que no es planteable dentro del paralelismo tal y como acabo de presentarlo-, o, por otro lado, se le supone un equivalente del lado de la extensión, algo que parece, igualmente, difícil. En las siguientes líneas abordaré esta cuestión sobre la que creo que recae el peso de esta controversia. Por ahora, puedo adelantar que la identidad de la idea con lo ideado es precisamente el propio modo, expresado en el atributo del pensamiento, idea e ideatum se fusionan en aquello que no está ya en otros atributos, sino que es todos ellos, puesto que su separación, empero, es mera retórica del pensamiento - de la misma manera en que un modo de la extensión también es un modo de otro atributo desconocido, expresado de otra forma-.

3 Cítese, por ejemplo, la clara y sencilla exposición de Errol Harris: «[...] la teoría no es un paralelismo o un dualismo psico-fisico [...], aunque es así como más frecuentemente se representa, presumiblemente porque Spinoza niega la interacción entre cuerpo y mente. La teoria es de identidad [...].» Cf. Errol Harris, The Substance of Spinoza, Humanities Press, Nueva Jersey, 1995, p. 62.

4 Por ejemplo, en E, I, 4, dem: «[...] nada hay fuera del entendimiento que sea apto para distinguir varias cosas entre sí, salvo las substancias o, lo que es lo mismo [...] sus atributos y sus afecciones.» (Cursivas mías).

5 E, II, 7, esc. 


\section{RECURSIVIDAD Y REFLEXIVIDAD DE LA IDEA DESDE LA PERSPECTIVA DEL PARALELISMO IDENTIFICATIVO}

El problema Relativo a las ideas que tienen por contenido otras ideas merece un debate, y su repuesta quizá no sea la definitiva en estas líneas, pero creo que existe una manera de enfocar esta temática sin poner en entredicho los cimientos ontológicos del sistema, y en este sentido puede servir adecuadamente como solución al mismo. Cuando una idea toma por ideatum otra idea, lo que, en esencia, es el fundamento de esta crítica a la amplitud del pensamiento en cuanto éste idea a otros modos y a sí mismo, aparentando salirse de sí o abarcar dos realidades diferentes, si ello es concebido desde la auténtica realidad de la identidad sustancial ya comentada, se comprenderá rápidamente que dicha idea de una idea debe ser también un modo en todos los infinitos atributos, lo que parece absurdo -la idea de un modo de la extensión constituye el mismo objeto en el atributo del pensamiento, de ahí esta identidad, pero ¿la idea de una idea de un modo de la extensión tiene un correlato con otro modo de la extensión? La idea de una idea, desde luego, no tiene por objetivo una idea vacía -pues entonces no habría nada que idear-, sino ese mismo modo de otro atributo.

Al decir que una idea $I$ de un objeto $x$ en otro modo-llámese I(x)-, es ideada por otra idea, llámese, $I_{2}$, entonces su estructura sería tal que $\mathrm{I}_{2}[\mathrm{I}(\mathrm{x})]$, esto es, la idea sigue teniendo por contenido su expresión modal en otro atributo -léase, sigue siendo expresión modal en el pensamiento de otro atributo-, y dicho contenido es sine qua non para la realidad de la idea, y sin él no hay idea posible. La expresión en cualquier otro atributo de lo que en el pensamiento será una idea es lo que he llamado $x$-en este caso, un modo de la extensión-, $I$ es la idea de $x$, que es $x$ mismo y nada diferente a ello más que en relación al intelecto,${ }^{6}$ pues son el mismo modo. Una idea del tipo $I_{2}$, entonces, es algo más que el objeto en cuanto extensión-pensamiento, esto es, en tanto cuerpo-idea, pues se trata de una idea que objetiva a la relación $\mathrm{I}(\mathrm{x})$ - un mismo modo, representado ahí en dos de los atributos en que se expresa-. $I_{2}$ consiste en una idea que recoge, reconoce u objetiva a ese modo, haciéndolo presente al pensamiento, esto es, a una interioridad pensante: dice algo más que evidenciar que todo lo extenso es representable también como idea, da el salto de la impersonalidad de la idea a otro tipo de pensamiento, a uno personal y consciente.

Hasta aquí creo que puede prolongarse esta reflexividad de la idea. A mi parecer, una idea que refiere a la idea de una idea $-\mathrm{y}$ cuantas se quiera prolongar-, del tipo $\mathrm{I}_{3}\left[\mathrm{I}_{2}[\mathrm{I}(\mathrm{x})]\right]$ sólo tiene realidad en el lenguaje, y no creo que tenga representación ontológica dentro del modelo spinozista. Es consecuencia lógica del sistema que cada idea es expresión en el atributo del pensamiento de un modo

6 supra, n. 4. 
en otros atributos, y también que, cuando se habla de ideas personales, esto es, dadas en una interioridad pensante, éstas hablan a una intelección consciente, y ello significaría que existe una idea que puede contener otra idea -una idea que sabe que hay ideas-; ahora bien, más allá de esto, una regresión ad infinitum me parece un mero artificio del lenguaje, sin ningún estatus en la realidad. Una idea del tipo anterior, esto es, $\mathrm{I}_{3}\left[\mathrm{I}_{2}[\mathrm{I}(\mathrm{x})]\right]$, no aporta nada a la identidad de una idea con su expresión en otro modo -lo que he expresado mediante la relación $\mathrm{I}(\mathrm{x})$-, y otra idea que objeta a dicha idea, esto es, el reconocimiento de la propiedad idea - una idea que trae a la consciencia la presencia en tanto idea de la identidad $\mathrm{I}(\mathrm{x})$, y que he llamado $\mathrm{I}_{2}[\mathrm{I}(\mathrm{x})]-$. Por ello, una idea del tipo $\mathrm{I}_{2}[\mathrm{I}(\mathrm{x})]$, que es idea de otra idea que tiene por contenido - que es-un modo expresado en otro atributo, es un fenómeno de la conciencia: cuando sabemos algo, sabemos que lo sabemos.

En el Tractatus de Intellectus Emendatione Spinoza ya se había pronunciado brevemente sobre esta recursividad de las ideas, cuando hablaba del método del conocimiento. Creo que estaría de acuerdo con lo anteriormente expuesto al afirmar respecto de dicho método lo siguiente:

A cuyo fin, lo primero que hay que decir es que no se producirá una búsqueda al infinito es decir, que para hallar el mejor método de investigar la verdad no se requiere otro método para investigar el método de investigar; y para investigar el segundo método no se requiere un tercero, y así al infinito, puesto que de ese modo no se lle $\neg$ garía nunca al conocimiento de la verdad o, mejor dicho, a ningún conocimiento.. ${ }^{7}$

Se enfatiza en esta obra, también, el absurdo de la reflexividad y regresión ad infinitum de las ideas, que no puede ir más allá de una idea primera y aquella que la objetiva, cuando afirma, otra vez respecto del método del conocimiento, que: «De ahí se desprende que el método no es más que el conocimiento reflexivo o la idea de la idea. Y como no hay idea de idea, si no se da primero la idea, no se dará tampoco método sin que se dé primero la idea.» ${ }^{8}$ Es cierto que el Tractatus no es aún la Ética, y que ciertas nociones evolucionaron, se transformaron o reexpresaron en otros términos en ella, pero creo que el germen de esta concepción puede hallarse ya en este primer opúsculo. Spinoza habla en él de las ideas: existe una idea de Pedro, que es algo real, y la idea de la idea de Pedro es también algo real -real en cuanto pensamiento y, presumiblemente, de alguna manera representable en términos de extensión-, ésta dice además que la idea de Pedro es la esencia objetiva de Pedro y, así, esta segunda idea es

7 TIE, 30. Utilizo, en ésta y las sucesivas citas al Tratado de la reforma del entendimiento, la traducción de Atilano Domínguez en Alianza, Madrid, 2006.

8 TIE, 38. 
algo distinto de Pedro - no es ya la identidad Pedro en extensión-pensamiento, sino la idea que revela la presencia de esa otra idea en una conciencia-. Aquí Spinoza, que aún no ha desarrollado con amplitud su ontología, su paralelismo ni su discurso ontológico, se refiere estrictamente al intelecto al decir que la idea de Pedro es algo distinto de Pedro, pues parece que Pedro, modo de la extensión con su representación en el lado del pensamiento, no es aquello que imaginamos cuando pensamos acerca de si sabemos que conocemos la idea de Pedro.

Sin embargo, con lo dicho anteriormente, cabe explicar para la disolución de esa peligrosa metáfora espacial que la idea de Pedro -esto es, el correlato directo de la intelección del lado del pensamiento que inextricable e indefectiblemente va unida al modo Pedro de la extensión-, en su presencia mental, mucho más vaga e imprecisa con la que, por ejemplo, evocamos su imagen cuando no está presente, no es más que la propia idea de Pedro en una versión más pobre, menos compleja $-\mathrm{y}$ que, además, puede darse en nuestra mente emponzoñada con otras ideas fragmentarias-. El artificio mental que supone elaborar una idea de Pedro debe ser la misma idea aunque, siendo más lejana -fuerzo la metáfora espacial conscientemente con el sentido de más vaga-, debe ser también más imprecisa e imperfecta -y en tanto consciente, se convierte ya en idea que pretende formar la idea de Pedro a partir de aquello que conoce de él en los atributos en que se le ha visto expresado-. En favor de esta opinión de la mayor realidad o cercanía de la proximidad de una idea con su referente real creo que puede citarse el siguiente pasaje también del Tractatus:

[...] para que yo sepa, no es necesario que sepa que sé. Y de aquí se sigue, a su vez, que nadie puede saber qué es la máxima certeza, sino aquel que posee la idea adecuada o esencia objetiva de alguna cosa; justamente, porque certeza y esencia objetiva son lo mismo. ${ }^{9}$

Ya en la Ética, estas reflexiones hablan estrictamente de ideas humanas, intelecciones que habitan una mente y que, en efecto, se identifican con sus respectivos modos en otros atributos, aunque muchas veces resulte difícil atisbar o comprender esta interrelación. En el escolio a la proposición vigésimo primera de la segunda parte, Spinoza, primero, unifica la idea del cuerpo con el cuerpo al afirmar: «[...] que la idea del cuerpo y el cuerpo, esto es [...], la mente y el cuerpo, son un solo y mismo individuo, al que se concibe, ya bajo el atributo del Pensamiento, ya bajo el atributo de la Extensión.» ${ }^{10} \mathrm{Y}$, respecto

9 TIE, 35.

10 Intervengo, en este punto y también en adelante, la traducción que hace Vidal Peña del término mens por alma, interpolando mente en su lugar. Vidal Peña, en 1975 -como también posteriormente Atilano Domínguez, en 2000-, se decantó por traducir mens como alma. El 
de la idea de la mente con respecto a la propia mente-que es, como ya se ha remarcado, también una idea-, afirma acto seguido que: «[...] la idea de la mente y la mente misma son una sola y misma cosa, concebida bajo un solo y mismo atributo, a saber, el del Pensamiento.» Aquí Spinoza habla acerca de la idea de una idea en términos de forma, al enunciar unas líneas más abajo que:

«[...] en realidad, la idea de la mente - esto es, la idea de la idea- no es otra cosa que la forma de la idea, en cuanto ésta es considerada como un modo del pensar sin relación con su objeto. En efecto, en cuanto alguien sabe algo, sabe sin más que lo sabe, y sabe a la vez que sabe lo que sabe, y así hasta el infinito.» ${ }^{11}$

Encontramos aquí, en efecto, que dada una idea del tipo $\mathrm{I}_{3}\left[\mathrm{I}_{2}[\mathrm{I}(\mathrm{x})]\right]$, la carga de ideas que objetiva otras ideas se muestra espuria en la medida en que lo fundamental de la idea, aquello que la disuelve, es la identidad elemental cuerpo-idea, ${ }^{12}$ e incluso la idea que tiene por objeto esa identidad es la forma de la idea, que puede extenderse lógicamente hasta el infinito, pero que no aporta nada a la relación elemental más que ese salto a la conciencia. Son ya muchos los autores que se han preguntado por esta unión entre lógica y psicología en Spinoza, toda vez que su explicación de los fenómenos mentales se subsume a una formulación lógica de relaciones entre ideas. No creo que el autor hispanoportugués estuviera en desacuerdo con que el desarrollo del conocimiento humano fuese completando todo aquello de lo que su filosofía no pudo dar cuenta por la supeditación a un contexto científico muy diferente - en la actua-

razonamiento concreto de Vidal Peña consistía en evitar cualquier concepción cerebralista que el término mens pudiera sugerir desde una lectura moderna ( $C f$. Ética, Alianza, Madrid, p. 96, n. 24). Esta cautela que manifestaba Vidal Peña en 1975 ha quedado, a mi juicio, superada en el tiempo por las actuales investigaciones neurocientíficas, de enorme complejidad y muy alejadas de cualquier positivismo cientificista. En mi opinión, de acuerdo con la ontología materialista de Spinoza, el término mens puede y debe ser traducido por mente sin mayores riesgos, distinguiéndolo así del término anima, o sea, alma, que es propio del dualismo de Descartes y frente al cual Spinoza marcó distancia en su obra. Sobre el término mens en la Ética de Spinoza, el estudio pionero de Emilia Giancotti: Sul concetto spinoziano di mens, en Giovanni Crapulli y Emilia Giancotti [eds.], Ricerche lessicali su opere di Descartes e Spinoza, Lessico intelletuale Europeo III, Edizioni dell' Ateneo, Roma, 1969. También sobre sobre la oportuna traducción de mens por mente, $C f$. Vicente Hernández Pedrero, reseña de la edición de Atilano Domínguez de Trotta, del año 2000, La Ética de Spinoza, en Laguna (Vol. 8), Universidad de La Laguna, La Laguna, 2001, pp. 205-206.

11 E, II, 21, esc.

12 Michael Della Rocca, en un artículo de 1993 titulado: Spinoza's Argument for the Identity Theory, defiende igualmente esta relación de identidad entre cuerpo e idea y la llama Identidad numérica (numerical identity). Cf. Spinoza's Argument for the Identity Theory, en Genevieve Lloyd (ed.), Spinoza: Critical assessments of leading philosophers [t. II], Routledge, Londres, 2001, pp. 140-164. 
lidad tampoco se podrían resolver estas dudas, ciertamente, aunque estemos más cerca de un mejor entendimiento del cerebro humano-. Joachim, autor ya citado en el epígrafe anterior, había señalado anecdóticamente en su estudio ${ }^{13}$ que, si el proyecto spinozista hubiera sido completado, éste comprendería otros tantos trabajos: si el Tractatus es una lógica a partir de la cual eliminar de la mente las formas erróneas de pensamiento, debería igualmente escribirse una Medicina para el cuerpo, una Teoría de la mecánica, una Física centrada estrictamente en el ámbito de la extensión, Teorías sobre moral y Estudios para la educación de los jóvenes Creo que, en efecto, Spinoza habría estado muy de acuerdo en continuar su proyecto de manera holista para con el resto de disciplinas del conocimiento -es notorio que el hispanoportugués tendió hilos en su Ética entre disciplinas que hoy se han separado más que unido, lo que debe ser una prueba razonable en favor de esta afirmación-.

En cualquier caso, la recursividad de la idea, que plantea un problema aparentemente más lógico que psicológico, encierra, como ya debe haberse desprendido con claridad, realmente el problema de la autoconsciencia, la idea reflexiva que se hace a sí misma objeto, como ha señalado el propio autor en la citada proposición anterior. Con todo, una idea $I_{2}$ que contiene a una de tipo $\mathrm{I}(\mathrm{x})$-donde, siguiendo la doctrina del paralelismo, $I$ constituye la expresión de $x$ y no son elementos diferenciados más que considerando $I$ como representación estrictamente mental en el ámbito del pensamiento-, tanto como una idea $I_{3}$ que sea idea objetiva de $I_{2}$, y así al infinito, revelan tan sólo el fenómeno de la conciencia de la idea. En efecto, $I$ seguirá siendo la expresión de $x$ en el modo del pensamiento aunque no haya un humano que la piense, pero cuando un humano puede acceder a $I$ o a $x$, entonces él es consciente de su idea de $I$, lo que, se asume, es una nueva idea que objetiva a $I$. La problemática sobre el absurdo de la regresión ad infinitum de la idea se simplifica al considerar que esta inagotable sucesión se reduce al fenómeno de la autoconciencia y, si esto es así, el planteamiento lógico de la cuestión puede trasladarse al ámbito de la psicología y abandonar la lógica.

Creo que es posible, en este punto, considerar a esa idea que objetiva a otra idea como la propia idea de autoconciencia; una idea que viene a romper esa sucesión regresiva ad infinitum que, aunque formal y lógicamente planteable, no tiene por qué hablar de objetos reales. En este sentido, en la idea compleja que es una mente humana -como reconoce Spinoza ${ }^{14}$-, una de ellas debe ser una idea capaz de objetivar otras ideas de una manera transparente y, del mismo modo que se tiene en la mente la idea de Pedro o de un planeta, debe haber una

13 Harold H. Joachim, op. cit., 4, n. 2.

14 E, II, 15: «La idea que constituye el ser formal [de la mente] humana no es simple, sino compuesta de muchísimas ideas.» 
idea dentro de esa mente como conjunto de ideas que es la que le habla o le revela la presencia de otras ideas. Esto, en cualquier caso, no clarifica el cómo de la idea, su porqué biológico, es decir, la aparente diferencia entre las ideas en-sí que no necesitan ser pensadas por nadie y, siguiendo la terminología de Vidal Peña, ${ }^{15}$ las ideas para-sí que, en este sentido, son pensadas y referidas a sí mismas. Lo importante es que, a mi juicio, el fenómeno de la autoconciencia puede representarse en el modelo de Spinoza en los mismos términos que cualquier otra idea del atributo del pensamiento -sobre su referente del lado de la extensión se hablará enseguida-, una idea que cumple los mismos patrones de identificación con su objeto, aunque su explicación definitiva deba esperar a una posible futura respuesta de la investigación científica -si es que una tal respuesta pudiera tener lugar-.

Un planteamiento similar a este respecto puede encontrarse en un artículo de David Savan. ${ }^{16}$ Su breve síntesis apoya la tesis que acabo de exponer, aunque su reconstrucción no es exactamente la misma, pues su distinción dota de estatus real a una sucesión de tres ideas, y no de dos, como la que he expuesto. Sea como fuere, su posición se mantiene en un sentido en los términos tratados: la idea del objeto a idear -lo que he llamado identidad idea-modo, o I(x)-, y la consciencia de dicha idea que de alguna manera es otra idea que hace de intermediaria entre el cuerpo externo y la mente, representándola dentro de la idea compleja que es esta última, algo que él ha separado en dos pasos y que yo he unificado, pues Savan añade esa tercera idea que re-presenta, advirtiendo de la presencia del objeto externo a la mente humana. Su exposición puede servir como marco de lo que ya ha sido expuesto en torno a la recursividad de la idea:

En representaciones de un cuerpo externo, tres ideas están implicadas. La idea A es la mente, que es la idea del cuerpo humano como un todo. B, la idea de la imagen, es un afecto de la mente y se subordina a ella. Es una idea compleja, que registra la presencia actual del agente externo que, en parte, da forma a la imagen. $\mathrm{C}$ es la idea de la presencia de B en A. Mientras que A y B son ideas cuyos objetos son cuerpos, $\mathrm{C}$ es una idea cuyo objeto es una idea de una relación de ideas. En tanto que B es en sí misma una idea de presencia, $C$ es la idea de la presencia de una presencia, y así representa. ${ }^{17}$

15 Vidal Peña, Eternidad y temporalidad 'en' Spinoza 'hacia' Hegel, en María Isabel Lafuente [ed.], Estudios sobre filosofía moderna y contemporánea, Universidad de León, León, 1984, pp. 57-76.

16 David Savan, Spinoza on Duration, Time, and Eternity en Genevieve Lloyd [ed.] op. cit., pp. 364-390.

17 David Savan, op. cit., p. 372. 
Creo más simple la anterior exposición entre la idea original que es identidad con otros modos y la idea de otra idea que permite señalar esta relación en la mente, pues no añade nuevos problemas a esta, ya de por sí, prolífica temática -en su versión cabría preguntar qué es, entonces, esa idea que está entre medio de la misma idea en cuanto modo y la mente, y que considera que tiene por contenido una relación de ideas-. Y, aun así, no ya a la exposición de Savan, sino a la que he defendido, resta por hacerle una pregunta central: ¿de qué otros atributos es expresión la idea que objetiva a la idea original? ¿Tiene un equivalente corpóreo del lado de la extensión? Creo que, en efecto, lo tiene, y es la estructura subyacente a un cerebro humano que le permite albergarla. Parece obligado, pues, considerar que esta $I_{2}$ según mi anterior esquema es, desde luego, identidad con la realidad estructural de un cerebro humano, ${ }^{18}$ pero del mismo modo en que la idea de Pedro es correlato de la estructura física, biológica, psicológica o química del mismo. La versión de Savan creo que tendría problemas para explicar-dentro del paralelismo identificativo-esa idea que él considera real y que está entre medio del modo ideado y la mente del sujeto pensante - que re-presenta-, pues atribuirle un referente del lado de la extensión, el único del que podemos hablar junto al pensamiento, resultaría problemático, si no imposible, y el paralelismo exige que cada elemento de cada atributo tenga presencia - esto es, sea algo en- otro atributo.

La forma de la idea - que constituye la idea de la mente, afirmaba Spino$\mathrm{za}^{19}$-, podría ser, en términos extensos, estructura; en el caso de un ser humano, la estructura de un cerebro que posibilita el conjunto de ideas que forman tal individuo, así como su posibilidad de conocer y conocerse - esto es, alberga esa idea que se vuelve sobre las demás, pudiendo ser esa idea la propia estructura global de un cerebro humano-. Lamentablemente, ni en época de Spinoza ni en la actualidad la psicología ni la neurociencia han podido responder aún a esta pregunta, con lo que adelantar una respuesta sería especular demasiado -el propio Spinoza tampoco lo intentó-. Creo que puede afirmarse que la problemática en torno al fenómeno de la autoconciencia-ahora expresado en términos de recursividad de ideas-, no compromete estructuralmente el modelo spinozista, así que puede dejarse en suspenso e integrarse en el mismo a medida que la ciencia indague sobre ello. Cito a Vicente Hernández a este respecto:

[...] la actual filosofía de la mente es deudora del spinozismo cuando busca determinar el carácter de la correlación entre la mente y el cerebro humanos. Epistemológicamente, la existencia natural y ontológica (no espiritualista) de esa correlación ha dejado de ser problemática; los problemas se dirigen ahora hacia la

18 Identidad que sólo aparece, por cierto, en comunión con otras ideas, puesto que sin algo que idear, no le sería posible a esta idea de autoconsciencia constituirse.

19 Cf. supra, n. 11. 
traducción en términos de explicación teórica del doble fenómeno mente-cerebro, al sentido causal del correlato evento mental-estado físico. ${ }^{20}$

\section{CONCLUSIONES Y NOTAS FINALES}

Como fue expuesto con anterioridad, la subordinación de ideas a ideas parece un artificio lingüístico formal, puesto que toda idea se identifica con un ideatum y, todo lo demás, con la salvedad del fenómeno de la conciencia, es girar en torno al mismo concepto. La idea de una idea de una idea de Pedro no creo que tenga representación alguna en la mente, más que juntando imágenes fragmentarias de Pedro y alejándolas o repensándolas en ideas separadas -hay que estar alerta, nuevamente, contra la metáfora espacial- que se representan o imaginan como seguidas unas de otras. Esto, y exceptuando, empero, la autoconciencia, que es otro fenómeno, creo que puede ser representado como una forma de unir ideas fragmentarias y nada más - una tendencia, es preciso señalar, propia del primer género de conocimiento-.

Por cierto que, hoy en día, con una ciencia más avanzada que la del XVII, podría hacerse el ejercicio inverso a la supuesta prevalencia del atributo del pensamiento sobre la extensión -haciendo una breve digresión retomando aquella cuestión-: podría intentar supeditarse el atributo del pensamiento al de la extensión -el reduccionismo fisicalista en cierta medida lo hace-; es decir, incluso la mente, las ideas, cualquier proceso mental, sobreviene de una naturaleza corpórea y, en este sentido, todo se reduciría a ella. El propio conocimiento de los fenómenos mentales ha sido pretendido por algunos a partir de una imagen reduccionista y fisicalista de lo real. El pensamiento no sería, pues, nada más que una forma sofisticada de materia extensa, y así esta extensión tendría primacía sobre aquél, si acaso no lo elimina. Errol Harris matizó, a colación de lo que comenté en la introducción de este artículo, ${ }^{21}$ lo siguiente:

La teoría es de identidad, pero no del mismo tipo que las teorías de la identidad neural que han sido propuestas por filósofos contemporáneos como Herbert Feigl, U. T. Place, y J. J. C. Smart. Estos escritores modernos niegan, en efecto, la existencia de ideas. ${ }^{22}$

No comparto, añado brevemente, una idea de este tipo: ni creo que el pensamiento tenga una posición privilegiada, como he mostrado, ni creo que la extensión la tenga, excepto que nos refiramos estrictamente a la identidad

20 Vicente Hernández Pedrero, Ética de la inmanencia: el factor Spinoza, Servicio de publicaciones ULL, La Laguna, 2011, p. 80.

21 supra, n. 3.

22 Errol Harris, op. cit., p. 62. 
entre todas las expresiones modales de todos los atributos, en cuyo caso por supuesto que todo lo real es extensión, y pensamiento, y así con todos los atributos. Deleuze, por citar sólo un ejemplo, ha explicitado esta idea que se colige directamente de las primeras formulaciones de la Ética:

Los atributos son realmente distintos: ninguno necesita de otro, ni de nada más, para ser concebido. Expresan así cualidades substanciales absolutamente simples; por eso, debe afirmarse que una substancia corresponde a cada atributo cualitativa o formalmente (no numéricamente). [...] La distinción real entre atributos es una distinción formal entre «quiddidades» substanciales últimas. ${ }^{23}$

Así pues, y al amparo de lo esbozado, el pensamiento en tanto atributo no se vería comprometido - ni comprometería-, la naturaleza del pensamiento humano expresado en términos de ideas de la manera en que ha sido recreado aquí. La asunción del paralelismo spinozista en tanto identidad permite a la Sustancia, expresada bajo el atributo del pensamiento, estar igualmente compuesta de modos que se organizan formando estructuras modales más complejas -verbigracia, una mente humana-, del mismo que, del lado de la extensión, un cuerpo humano es una estructura superior a cada uno de sus átomos constituyentes. Tras el rechazo de una posible regresión ad infinitum de ideas y, junto a ello, la mantención del paralelismo-identidad en todo momento respecto del componente mental del ser humano, el atributo pensamiento y todo aquello expresado bajo su alcance no ostentaría una inaceptable primacía en la configuración de la Sustancia, bien sea por esa supuesta capacidad de transgredir sus propios límites y abarcar al resto de atributos, cristalizada en la forma de aquella metáfora espacial, o bien mediante los problemas relativos al pensamiento que se piensa a sí mismo y cómo éste se integraría dentro del propio marco de su atributo y sus consecuencias.

Volviendo a la temática en torno a la configuración interna del atributo del pensamiento -evitando incurrir en aquella capciosa metáfora espacial-, y con objeto de recuperar aquella cuestión por la recreación mental de la idea de Pedro, uno podría preguntar, también, dónde se encuentra la idea completa de Pedro, que es la que más claramente concebimos, supuestamente, al tener a Pedro delante; no obstante, y como es obvio, la representación física de Pedro es un modo de la extensión, y su expresión en cuanto pensamiento es otra cosa, algo que se revela cuando Pedro interactúa cognitivamente con otro o cuando desarrolla una conducta. La expresión de Pedro en términos de intelección es, se dirá, incompleta, siempre velada por aquello que Pedro muestre, y ello es cierto; pero también el conocimiento de la expresión en cuanto extensión de Pedro es a su vez fragmentario y limitado, ya que el ser humano sólo puede

23 Gilles Deleuze, Spinoza: filosofía práctica, Tusquets, Barcelona, 2003, p. 66. 
acceder a los modos dentro de sus capacidades - tan limitado es que sólo puede comprender y atestiguar dos de los infinitos atributos-. Ver el cuerpo de Pedro es ver una limitada forma de expresión de Pedro en cuanto extensión, pues Pedro es algo más que sus miembros, es todos los átomos y partículas subatómicas inaccesibles a nuestros sentidos -como Pedro del lado del pensamiento, a nuestra inmediata percepción, no llega a presentarse con su inconsciente y sus otros tantísimos procesos mentales-. La ciencia ayuda a comprender y esclarecer modalmente los elementos del universo, en la medida de sus también limitadas capacidades; así, revela que el modo de la extensión Pedro es un organismo mucho más complejo y con más partes de las que a simple vista pueden constatarse, y de este modo la psicología revela que los procesos mentales de Pedro van más allá de su conducta inmediata, pero esto es otro tema.

Respecto de cómo es posible que un recuerdo de Pedro -fragmentado y limitado-, pueda ser idéntico a cierta representación -también fragmentada y limitada- de un Pedro que no está presente - que puede estar a cientos de miles de kilómetros de distancia-, no creo que necesite mayor explicación en términos del sistema: el Pedro físico puede estar todo lo lejos que sea, pero en el ámbito del pensamiento la distancia es inconcebible y absurda. Si del lado de la extensión aproximarse a Pedro es acercarse físicamente a él, del lado del pensamiento sería formar una imagen cada vez más clara y precisa del mismo, lo que, por supuesto, y aunque pueda estar ayudado por la presencia corpórea de aquello que se ha llamado Pedro, no le necesita en términos de extensión.

En cualquier caso, creo que la crítica en torno a la mayor extensión del atributo del pensamiento está anclada, como comenté desde el inicio de este trabajo, en el abuso de una metáfora espacial; por otro lado, y como espero haber mostrado a lo largo del mismo, el fenómeno de la conciencia y la posibilidad del pensamiento de hacerse objeto tanto a sí mismo como a otros atributos no debería, en ningún caso, otorgarle primacía alguna. Bajo la concepción del paralelismo en tanto identidad -la única que considero viable y la que Spinoza afirmó sin ambages-, y tomando a la conciencia como una idea cuya expresión en términos de extensión debe tener las características que tiene la estructura de un cerebro humano - con toda su complejidad-, no hay cabida a una mayor extensión del atributo del pensamiento, y por tanto los atributos de Dios que conocemos pueden llamarse, sin menoscabo para ninguno de ellos ni para el sistema entero, paralelos e idénticos en la Sustancia.

Daniel Álvarez Montero es Doctor en Filosofía por la Universidad de La Laguna

Lineas de investigación:

Filosofía moral, Filosofía de la ciencia, Ontología 
Publicaciones recientes:

«Metafísica de la Naturaleza en la filosofía de Spinoza: una interpretación del conatus como primera determinación ontológica de la Natura naturata» en Ágora: papeles de filosofía, 2017, n⿳36/1, pp. 203-221.

«Ontología y física en la filosofía de Spinoza: un nuevo marco cosmológico para la Natura naturata» en Éndoxa: Series Filosóficas, 2017, n³9, pp. 81-99.

Correo electrónico: astral.alv@gmail.com 